\title{
COMBINED CHANNEL ALLOCATION TECHNIQUES IN MOBILE RADIO SYSTEMS
}

\author{
Ailton Akira Shinoda \\ Research and Development Center - CPqD - Telebrás, Brasil \\ Michel Daoud Yacoub \\ University of Campinas, Brasil
}

\begin{abstract}
RESUMO: Técnicas globais de alocação compreendendo as várias formas de alocação dinâmica de canais -, e técnicas locais de alocação - caracterizadas pelos algoritmos que levam em conta o tráfego disponível nas áreas de sobreposição entre células -, são combinadas em um algoritmo comum para aplicações em sistemas rádio móvel. A análise de desempenho é levada a cabo através de simulação de Monte Carlo tendo como sistema sob investigação uma rede celular real. Os resultados mostram um desempenho notável de tráfego, substancialmente melhor do que aquele obtido ao se aplicar as técnicas individualmente. Um aumento da complexidade devido à combinação é certamente esperada, mas, devido a que as técnicas locais são razoavelmente simples de serem implementadas, acreditase que a complexidade global será ditada principalmente pela técnica global.
\end{abstract}

ABSTRACT: Global assignment techniques - comprising the various forms of the dynamic channel allocation algorithms -, and local assignment techniques characterised by the algorithms taking advantage of the traffic available at the overlapping service areas -, are combined into a common algorithm for mobile radio applications. The performance analysis is carried out by means of the Monte Carlo simulation having a real cellular network as the system under study. The results show a remarkable traffic performance, substantially better than that attained by the individual techniques. An increase in the complexity due to the combination is certainly expected, but, because the local techniques are reasonably simple to implement, it is believed that the overall complexity is mostly dictated by that inherent to the global technique.

COMBINED TECHNIQUES FOR CHANNEL ALLOCATION ALGORITHMS IN MOBILE RADIO SYSTEMS

Keywords: Wireless communications, Channel allocation algorithms, Mobile system performance.

\section{INTRODUCTION}

A great many channel assignment techniques, aiming at mobile radio systems applications, have been proposed and extensively investigated in the literature [1-10], as an alternative to the Fixed Channel Allocation (FCA). The researches in this area seem to have been intensified lately with the advent of the Personal Communications Systems. The contend TDMA versus CDMA has also contributed with a great share in this, because channel allocation is certainly a factor that can be used to improve the system traffic performance.

In general, the channel allocation techniques can be grouped into two classes: Global Assignment Techniques (GAT) and Local Assignment Techniques (LAT). The GAT group contains the various forms of Dynamic Channel Allocation (DCA), in which an arbitrary proportion of the channels available within the spectrum are assigned on a demand basis, according to the instantaneous or mean traffic behaviour. The LAT group is characterised by alternative routing strategies, the Directed Retry [9] being its best known representative, in which the routing procedures take advantage of the traffic available at the overlapping areas of adjacent cells.

The performance comparison of these techniques have been assessed within their own group and no, or little, attempt has been made to compare techniques belonging to different groups. In fact, the fundamental approach of the algorithms belonging to one or another group is rather dissimilar leading to a (mis)conception of exclusiveness, i.e., a decision being made in favour of one group already excludes the use of the other.

This paper proposes the conciliation of the two distinct factions by combining Global Assignment Techniques and Local Assignment Techniques into a common algorithm.

\section{GLOBAL ASSIGNMENT TECHNIQUES}

The algorithms belonging to the GAT group require a substantial amount of data processing and have a performance varying according to the traffic distribution. In particular, they perform less satisfactorily than the fixed allocation for high load [1-4] because of the spatial inefficiency caused by the channel locking, a strategy used to keep the cochannel interference within acceptable limits by keeping the required minimum reuse distance [1]. 
Among the infinitude of global assignment algorithms available in the literature we chose to analyse some well known techniques, namely, Borrowing Channel Allocation (BCA) [3, 4] and Hybrid Channel Allocation (HCA) [1], presented in their simplest form. The aim is not to analyse the techniques individually, but to illustrate the combination, thence assessing its performance. It is anticipated that more complex techniques can be used for the combined algorithm.

\subsection{Borrowing Channel Allocation}

In Borrowing Channel Allocation the channels are assigned to the cells on a fixed basis. At the arrival of a new call a cell, having all of its channels busy, looks for a free channel in the neighbouring cells. If more than one cell is found to be eligible for this purpose a channel is chosen out from the cell presenting more free channels. Call reshuffle can be implemented so as to diminish the spatial inefficiency caused by channel locking. In such a case a borrowed channel is returned to its original cell as soon as a channel, to which this call is reshuffled, is made available within the borrowing cell.

In a more recent work $[7,8]$ (Channel Borrowing Without Locking: CBWL), evolved from the borrowing algorithm, the channel locking impairment is solved by reducing the transmitted power of the borrowed channels, thence preventing the increase of cochannel interference. Because of the reduced power, these channels can be accessed only in part of the borrowing cell, which implies a signal sensing procedure to determine whether or not a mobile is in a region where the borrowing process can take place.

\subsection{Hybrid Channel Allocation}

In Hybrid Channel Allocation a portion of the channels is assigned on a fixed basis and the remaining portion on a dynamic basis. According to the proportion $D / F$ of dynamic $(D)$ to fixed $(F)$ channels, this technique may vary from the full dynamic allocation to the fixed allocation.. Given a ratio $D / F$ an increase in the channel usage can be attained by means of call reshuffle. The aim is to make more dynamic channels available so that the system can cope better with the sudden variation of the traffic. This is achieved by transferring calls using dynamic channels to fixed channels.

\section{LOCAL ASSIGNMENT TECHNIQUES}

The algorithms belonging to the LAT group identify a call as pertaining to two streams of traffic: a) fixed traffic, arising in the core of the cell and either carried via the corresponding base station or blocked; b) flexible traffic, arising near enough the boundary to be carried via one of the base stations to which it may have access.

Several local assignment techniques are available in the literature [9-12]. Among the strategies belonging to the LAT group we chose to analyse the Blocking Threshold Variation (BTV) [11, 12], for its versatility.

The BTV can be thought of as a generalised Directed Retry. In Directed Retry the decision to look for a second option cell to serve a flexible call is taken when the first option cell finds all of its channels busy. In BTV this decision is based on a threshold $T, T \geq 0$, chosen as a design parameter. Flexible calls are diverted to the second option cell in case the number of busy channels in their own cell exceeds $T$ and the second option cell has a number of free channels greater than that of the first option cell. Note that if $T$ is chosen to be $T>N$, where $N$ is the total number of channels in the cell, the flexible calls will remain in their own cell. Note also that BTV and Directed Retry are coincident for $T=N$. Moreover, if $T=$ 0 the flexible calls will always be diverted to the cell presenting more free channels. In this case the routing procedure coincides with that explored in the strategies Instantaneous Adaptation (IAP) and Instantaneous and Mean Adaptation (IMA), both analysed in Reference 10. The BTV strategy can also be applied to those calls already in progress which have been identified as flexible. This requires a call reshuffle procedure in which, based on the BTV decision rules, the flexible call is transferred to another channel, belonging to the neighbouring cell, so as to accommodate a non flexible call arising in the first cell.

\section{COMBINED TECHNIQUES}

The application of the algorithms belonging to the GAT group implies a change in the usage pattern of the channels with a consequent increase in the complexity of the network management. In the LAT case, although the rearrangements are purely local, some change in the usage pattern of the channels is also expected, but the application of the strategies does not imply any complex global network control.

The FCA is known to be simple to implement, to provide the maximum spatial efficiency in channel reuse, and not to be able to cope with the sudden variation of the traffic pattern. The GAT, in general, are known to render the system more adaptable to the variation of the traffic profile, to be complex to implement, and to be spatially less efficient. Because of this spatial inefficiency, as the traffic increases, the algorithms belonging to the GAT group are outperformed by the FCA. Certainly such an outperformance may be minimised by increasing the complexity of the allocation techniques. The algorithms belonging to the LAT group, by their turn, are known to be simple to implement, to always perform better than the FCA, and not to affect the spatial efficiency of the channel reuse but, certainly, at the expense of the increase in the interference levels $[13,14]$.

This paper proposes the combination of the Global Assignment Techniques and the Local Assignment Techniques to increase the traffic performance of the mobile radio system. For the algorithms belonging to the 
GAT, in which only a portion of the channels are assigned on a fixed fashion and the other portion on a dynamic fashion, the algorithms belonging to the LAT are applied fraightforwardly to the fixed portion. If only dynamic thannels are available the LAT can still be used but now aiming at the locked channels. In such a case, a channel focked in a cell can be used in a neighbouring cell by a mobile station located near enough the boundary of the two concerned cells. Note that a further combination of the latter and the former strategies is still possible, i.e., if a portion of fixed and dynamic channels is available the LAT can be applied aiming at both the fixed portion, as hormally used, and the dynamic portion, for the locked ehannels. However, in this paper the application of the local assignment technique is restricted to the fixed portion only.

\section{TRAFFIC MODEL}

The combination of Global Assignment Techniques and Local Assignment Techniques in a mobile radio system characterises a traffic process with some mutual traffic dependence and can be modelled by different means. Analytical methods are certainly impracticable if a large network, with any traffic distribution, and some interesting features, such a call reshuffle, are to be accommodated in the combined scheme. Monte Carlo simulation is doubtlessly a handy tool that can be used to more easily tackle the problem, and this is what has been utilised here. In our analysis we assume the following: a) traffic is Poisson; b) holding time has a negative exponential distribution; c) flexible calls occur with a given probability; and d) flexible calls may be routed to either cell as required.

\subsection{Performance Parameters}

The performance of the system will be analysed in terms of the following parameters:

$$
\begin{aligned}
& \text { System total traffic - } A \\
& \text { Proportion of flexible traffic of cell } i-\gamma_{i} \\
& \text { Imbalance of traffic of cell } i-\beta_{i} \\
& \text { Number of channels of cell } i-N_{i} \\
& \text { Blocking threshold of cell } i-T_{i}
\end{aligned}
$$

These parameters will be better characterised in the next subsection.

\subsection{Traffic Streams}

Consider an $n$-cell mobile radio system. For each cell $i, i$ $=1, \ldots, n$, define $A_{i}$ as its total traffic, $A_{\varphi i}$ as its fixed traffic, and $A_{\gamma i}$ as its flexible traffic. It is clear that

$$
A_{i}=A_{\varphi i}+A_{\gamma i}
$$

Because

$$
A_{\gamma i}=\gamma_{i} A_{i}
$$

then

$$
A_{\gamma i}=\frac{\gamma_{i}}{1-\gamma_{i}} A_{\varphi i}
$$

Assume that each cell contributes to the total flexible traffic of the system with the same proportion $\gamma_{i}=\gamma, i=1$, $\ldots, n$. Therefore

$$
\sum_{i=1}^{n} A_{i p}=(1-\gamma) A
$$

Let $A_{m}$ be the system mean traffic such that

$$
A_{m}=\frac{1}{n} \sum_{i=1}^{n} A_{i}
$$

Define the traffic imbalance of cell $i$ as

$$
\beta_{i}=\frac{A_{i}-A_{m}}{A_{m}}
$$

From Equation 6 it is readily seen that

$$
-1 \leq \beta_{i} \leq n-1
$$

We observe that $\beta_{i}=-1$ implies no traffic in cell $i$. whereas $\beta_{i}=n-1$ implies all the traffic in cell $i$. The condition $\beta_{i}=0$ signifies the balanced traffic situation. Again, from the definition of the imbalance parameter it follows that

$$
\sum_{i=1}^{n} \beta_{i}=0
$$

These equations can be manipulated to give

$$
A_{\text {ip }}=\frac{1}{n} A(1-\gamma)\left(1+\beta_{i}\right)
$$

In the same way

$$
A_{\gamma i}=\frac{1}{n} A \gamma\left(1+\beta_{i}\right)
$$

Let $b_{i}$ be the number of neighbouring cells touching the boundaries of cell $i$. This defines the number of effective boundaries of cell $i$. We note that in an infinite cellular 
network $b_{i}=6$, but $0 \leq b_{i} \leq 6$ in a real cellular system. Assuming the flexible traffic of cell $i$ to be uniformly distributed among its effective boundaries then the flexible traffic for each border is $A_{\gamma i} / b_{i}$. This assumption imposes a condition such that subscribers situated in the inner cells and those located in the cells of the periphery may have equal chance to access a second base station. This can be made possible by appropriately setting the system transmission quality parameters as required. In fact, it has been seen [15] that such a consideration is reasonably irrelevant for a large network, as is the case. The number of effective boundaries $b_{i}$ can be set to 6 for all of the cells and the final result is satisfactorily the same. Defining $A_{i j}$ as the flexible traffic available between the neighbouring cells $i$ and $j$, then

$$
A_{i j}=\frac{A_{\gamma i}}{b_{i}}+\frac{A_{\gamma j}}{b_{j}}
$$

Or, equivalently

$$
A_{i j}=\frac{1}{n} A \gamma\left(\frac{1+\beta_{i}}{b_{i}}+\frac{1+\beta_{j}}{b_{j}}\right)
$$

Given the parameters defined previously the traffic streams are then generated according to the traffic process already described.

\subsection{Performance Measure}

The performance of the system will be assessed by the blocking probability averaged over all of the traffic streams. This defines the system mean blocking probability $B_{m}$ such that

$$
B_{m}=\frac{\sum_{i=1}^{n} A_{i} B_{i \varphi}+\sum_{i=1}^{n} \sum_{j=1}^{n} A_{i j} B_{i j}}{A}
$$

where $B_{\varphi i}$ is the blocking probability of $A_{\varphi i}$ and $B_{i j}$ is the blocking probability of $A_{i j}$

As a function of the traffic parameters

$$
B_{m}=\frac{(1-\gamma) \sum_{i=1}^{n}\left(1+\beta_{i}\right) B_{i q}+\gamma \sum_{i=1}^{n} \sum_{j=1}^{n}\left(\frac{1+\beta_{i}}{b_{i}}+\frac{1+\beta_{j}}{b_{j}}\right) B_{i j}}{n}
$$

Note that $A_{i j}$.and $B_{i j}$ are only defined for neighbouring cells, which implies a maximum of six possible products of the kind $A_{i j} B_{i j}$, for each $i=1, . ., n$.

\subsection{Validation of the Model - heckpoints}

One of the relevant points to be considered in an investigation is how to validate the model that has been adopted. As far as this work is concerned the traffic model used here matches with the well accepted models utilised in similar works available in the literature. Therefore, the main question to be pondered is on the validation of the simulation results. Some checkpoints can be used for such a purpose.

Consider the BTV strategy alone. In this case if $\gamma=0$ (no flexible traffic) the algorithm coincides with FCA and the Erlang-B formula applies. In the same way, if $T_{i}>N_{i}, \forall i$ $=1, \ldots, n$, the strategy is never applied (it coincides with FCA) and again the Erlang-B formula can be used.

Consider the combined technique. As $\gamma$ diminishes the effects of the LAT in the combination tends to be less perceptible and the corresponding performance degrades into that of the respective GAT. In the limit when $\gamma=0$ the combined technique reduces to the appropriate GAT (HCA or CBA, as explored here). The same phenomenon occurs for $T_{i}>N_{i}, \forall i=1, \ldots, n$. Other checkpoints include the following. By decreasing the ratio dynamicchannels/fixed-channels the effect of the association HCA-BTV will tend to be that of BTV alone. The same approach, with a consequent similar effect, can be carried out with respect to the total number of channels allowed to be in the borrowed condition in the association CBABTV. In both cases, in the limit, when the corresponding parameter is set to zero, the combined techniques reduce to $\mathrm{BTV}$ alone.

\subsection{System Under Investigation}

The performance of the combined technique has been assessed in several network configurations with distinct traffic distributions [15]. In particular, the results to be shown here consider a cellular layout as depicted in Figure 1. This system is based on a real network [16] in which the 41 cells are grouped in a 7-cell pattern fashion, A, B, C, D, E, F, and G denoting the channel group. The traffic distribution is shown in Figure 1, in terms of the traffic imbalance parameter, as lines rising from the cells. A vertical solid line signifies a positive imbalance whereas a negative imbalance is pictured by a vertical dashed line. The initial total system traffic is considered to be 250 erl. and channels of the cells have been determined so as to have a mean blocking probability of $1.3 \%$.

\section{RESULTS}

The system mean blocking probability will be shown as a function of the proportion of the fiexible traffic for a given traffic load, and as a function of the traffic load for a given proportion of flexible traffic. The combined techniques considered here are BCA-BTV and HCA$\mathrm{BTV}$. In the fully association BCA-BTV there is not limit in the number of channels allowed to be in the borrowed condition (only the reuse distance constraint is taken into account). In HCA-BTV a proportion of $70 \%$ of fixed channels and $30 \%$ of dynamic channels is used. Several combinations, including DCA (totally dynamic)-BTV, CBWL-BTV, and others have been analysed in Reference 15 and the qualitative results confirm those to be shown here. Although the focus of this paper is on the combined techniques, it is certainly informative to show the performance of each one of their components (CBA, 


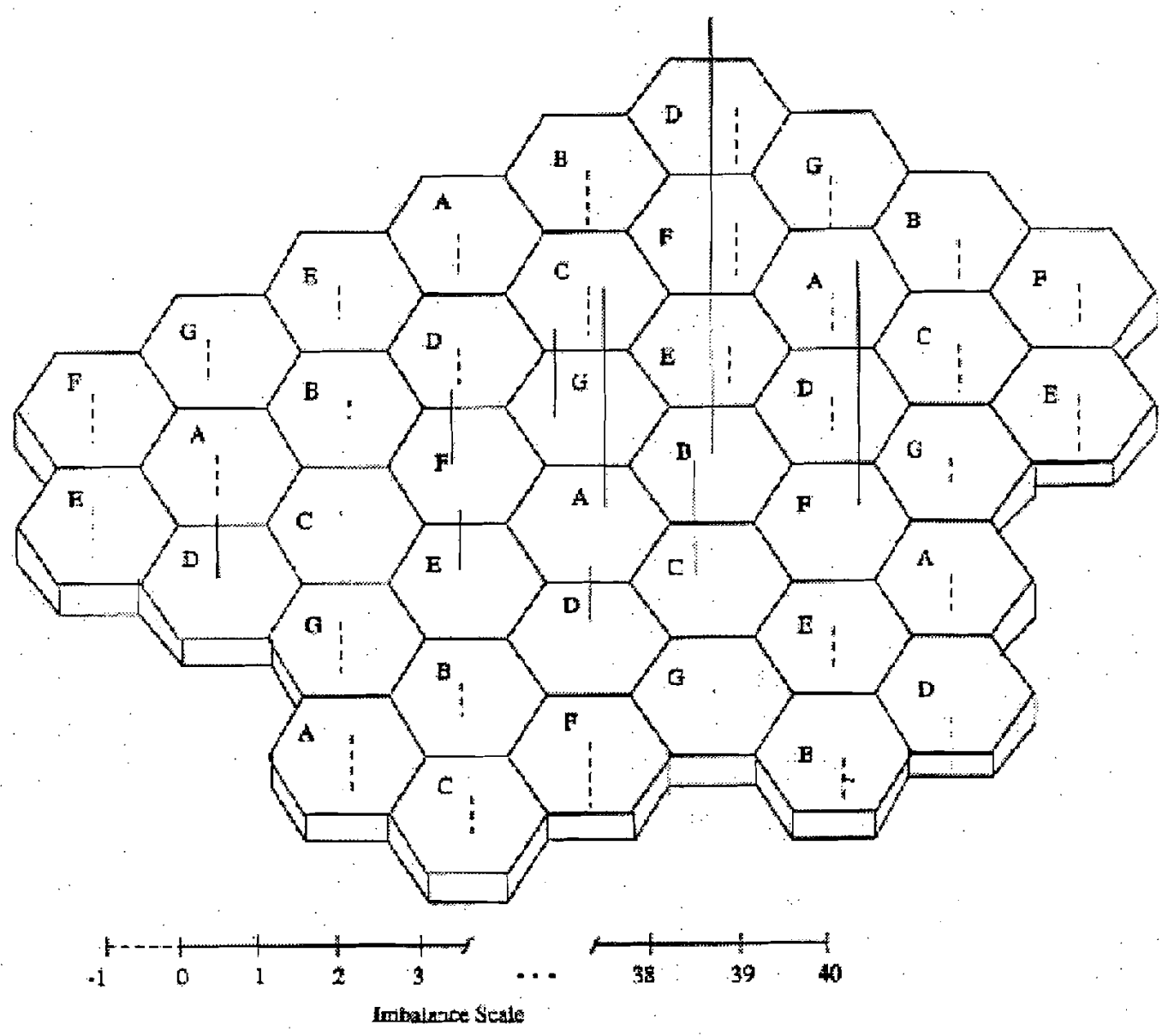

Figure 1: Cellular system and traffic profile shown in terms of the imbalance of traffic. Lines rising from the cells show the imbalance: solid lines signify positive imbalance; dashed lines signify negative imbalance. In a 41-cell system the imbalance may vary from -1 to 40 . In this system it does not exceed 7.

HCA, and BTV, as explored here) so as to investigate the effects of the combination.

\subsection{Mean Blocking Versus Proportion of Flexible Traffic}

The mean blocking probability as a function of the proportion of flexible traffic is shown in Figures 2 and 3 for CBA-BTV and HCA-BTV, respectively, given a total traffic of 1.6 times the initial traffic. The special cases, obtained by means of an appropriate manipulation of the parameters, in which the combined techniques reduce to each one of the individual techniques, are also shown.

Refer to the set of curves $a$, in Figures 2 and 3, for the combined technique degrading into BTV alone [11]. Note that the mean blocking probability is obviously insensible to the variation of $\gamma$ if $T_{i}>N_{i}$, in which case BTV coincides with FCA with the flexible calls being served by their own cells.. This concurrence is also observed for $\gamma=$ 0 , as expected. It can be seen that, in the absence of call reshuffle, there is a remarkable traffic performance improvement as the threshold $T_{i}$ goes from $T_{i}=N_{i}$ (directed retry) to $T_{i}=0$. On the other hand, the gain is essentially the same for values of threshold $T_{i}$ below $80 \% N_{i}$. Therefore, in practical terms, a system where the cells operate with a threshold as high as $80 \% N_{i}$. exhibits basically the same performance as that with cells operating with a nil threshold. The use of call reshuffle provides a substantial traffic gain but the variation effect of the threshold parameter is negligible.

Refer to the set of curves $b$, in Figures 2 and 3, for the fully combined techniques. The mean blocking probability is obviously insensible to the variation of $\gamma$ if $T_{i}>N_{i}$, in which case the combined technique coincides with the appropriate GAT (CBA in Figure 2 and HCA in Figure 3). This is also observed for $\gamma=0$, as expected. It is clearly noted that the gain provided by the combination is substantial. The mean blocking probability decreases steadily with the increase of $\gamma$. The decrease of $T_{i}$ also has a contribution in this, but in the absence of call reshuffle. The influence of the variation of $T_{i}$ is rather imperceptible in case call reshuffle is implemented. Moreover, call reshuffle itself does not play any significant role in the combined technique, if a reasonable value of $T_{i}$ (say $T_{i}=$ $80 \% N_{i}$ ) is adopted. In other words, the performance of the combined technique for an adequately chosen blocking threshold is essentially the same as that in which call reshuffle is implemented for any value of blocking threshold. 


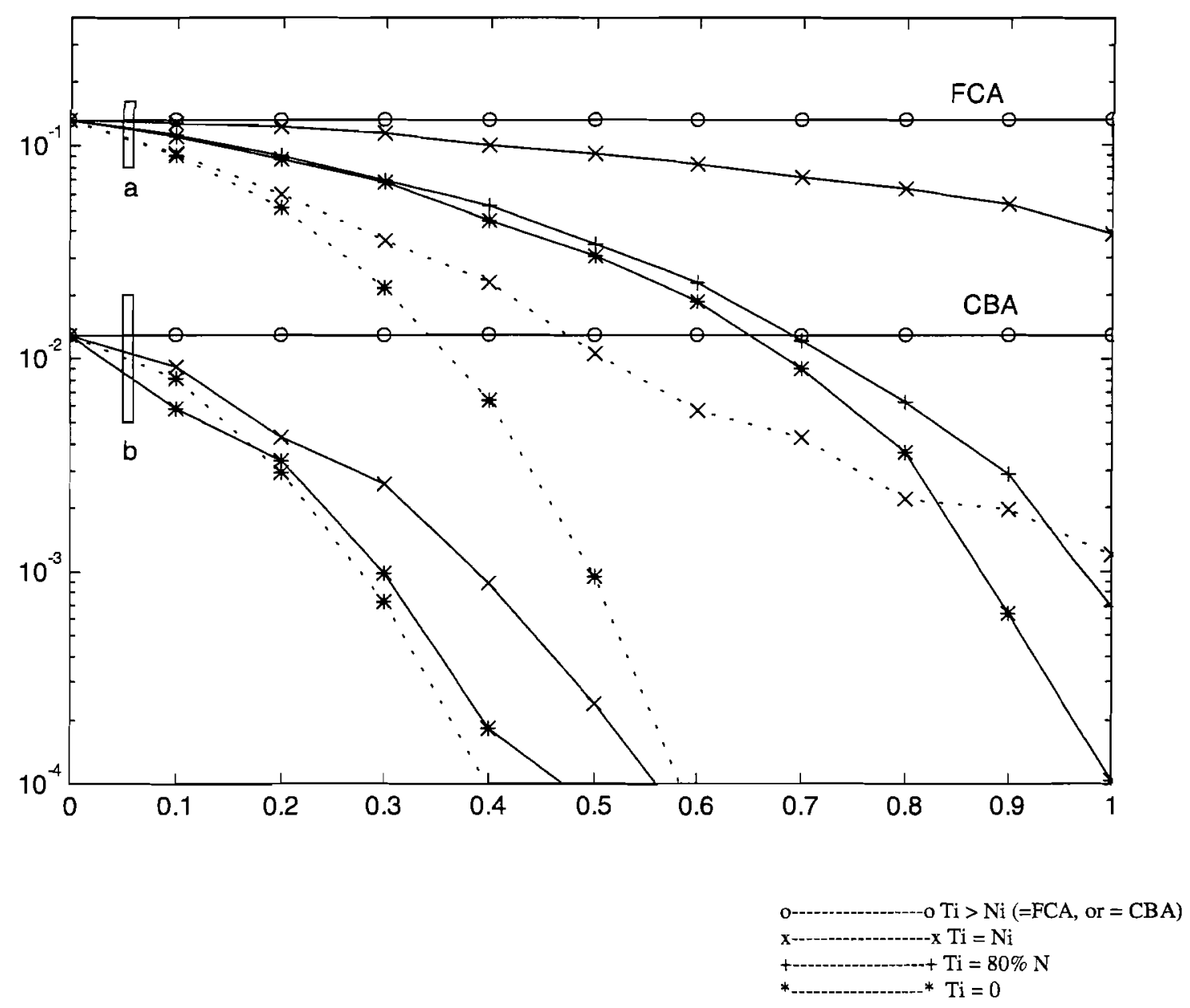

Figure 2: CBA-BTV : Mean blocking probability versus proportion of flexible traffic. Solid lines represent no reshuffle; dashed lines represent reshuffle.

a) Channels are not permitted to be the borrowed (CBA-BTV coincides with BTV)

b) Channels are permitted to be the borrowed (CBA-BTV is fully implemented)

\subsection{Mean Blocking Versus Normalised Traftic}

The mean blocking probability as a function of the traffic, normalised with respect to the initial traffic, is shown in Figures 4 and 5 for CBA-BTV and HCA-BTV, respectively, given a proportion of flexible traffic of $40 \%$.

The same comments of the previous section are also applicable here. In particular we stress the influence of the variation of the blocking threshold in the call reshuffle case and its irrelevance in the reshuffle application.

\section{SOME IMPLICATIONS OF THE COMBINATION}

The association of algorithms belonging to the GAT and LAT groups seems to provide a remarkable traffic gain, considerably higher than that obtained if the single techniques are used individually. A straightforward implication of using a compound algorithm is obviously the increase of the implementation complexity. Nevertheless, in general, the algorithms belonging to the LAT group are simple to implement because they deal with a purely local channel rearrangement. They explore the overlapping areas of cells and only the right to use the channels is transferred from one base station to the other. On the other hand, those algorithms in the GAT group deal with more complex procedures and with channel transfer itself. Therefore, although the decision rules of the combined technique become more elaborate, the overall complexity is predominantly dictated by that of the GAT algorithm.

Because of the use of a LAT strategy an increase of the interference levels, both for adjacent channel [13] and cochannel [14] interferences, can be expected because channels with marginally acceptable transmission quality may be utilised.

\section{CONCLUSIONS}

Combined global and local channel assignment techniques have been proposed and analysed. The global techniques 


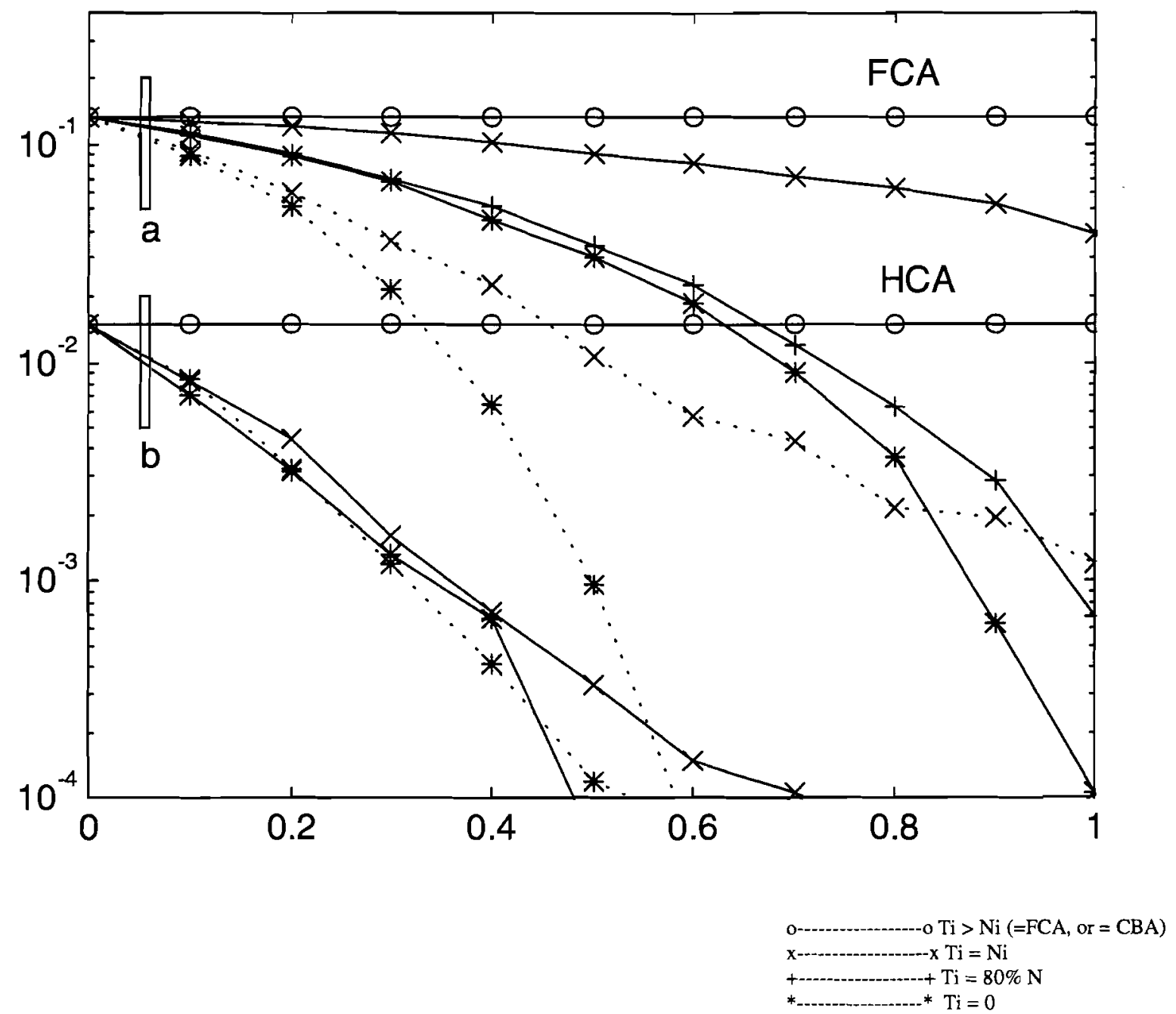

Figure 3: HCA-BTV: Mean blocking probability versus proportion of flexible traffic.

Solid lines represent no reshuffle; dashed lines represent reshuffle.

a) Dynamic-channels/fixed-channels = 0 (HCA-BTV coincides with BTV)

b) Dynamic-channels/fixed-channels $=3 / 7$ (HCA-BTV is fully implemented).

comprise the various forms of the dynamic channel allocation algorithms, whereas the local techniques are characterised by the algorithms taking advantage of the traffic with adequate communication with more than one base station, available at the overlapping areas between cells. In particular, this work explored a simple form of both Channel Borrowing Allocation and Hybrid Channel Allocation, representing the global techniques, and Blocking Threshold Variation, a generalised form of Directed Retry, representing the local techniques.

A Monte Carlo simulation study has been carried out, having a real cellular network as the system under investigation. The results show that the combined techniques provide a remarkable traffic performance, substantially better than that attained by the individual techniques. An increase in the complexity due to the combination is obviously expected, but, because the local techniques are reasonably simple to implement, it is believed that the overall complexity is mostly dictated by that inherent to the global technique. Furthermore, an augmentation in the interference levels is also anticipated, due to the application of local techniques, in which channels with marginally acceptable transmission quality can be used. On the other hand, a blocking threshold can be set so as to discipline the use of these channels. It was shown that a blocking threshold $T_{i}$ as high as $80 \% N_{i}$, where $N_{i}$ is the number of channels in cell $i$, yields a performance reasonably close to that obtained for $T_{i}=0$, in which case the use of these channels is indiscriminate. A higher threshold keeps more flexible calls within their own cells, and a better transmission quality is experienced

\section{REFERENCES}

[1] D. C. Cox and D. ' Reudink: "Increasing channel occupancy in large-scale mobile radio systems: Dynamic channel reassignment", IEEE Trans. On Comm., Vol. COM-21, No. 11, Nov. 1973, pp. 13021306. 


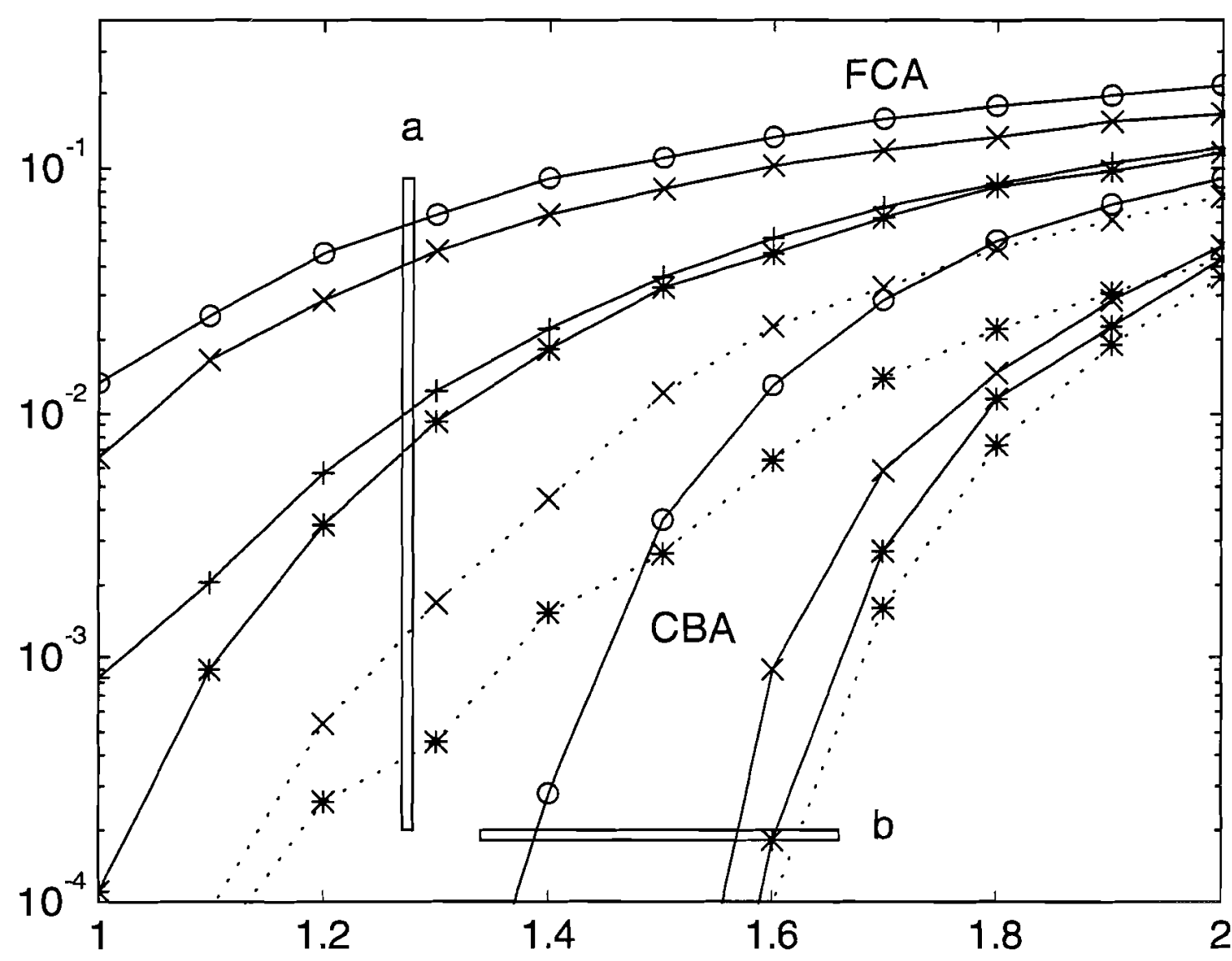

Figure 4: CBA-BTV: Mean blocking probability versus traffic.

Solid lines represent no reshuffle; dashed lines represent reshuffle.

a) Channels are not permitted to be the borrowed (CBA-BTV coincides with BTV)

b) Channels are permitted to be borrowed (CBA-BTV is fully implemented)

[2] J. S. Engel and M. M. Peritsky: "Statistically-optimum dynamic server assignment in systems with interfering servers", IEEE Trans. Veh. Technol., Vol. VT-22, No. 4, pp. 203-209, Nov, 1973.

[3] L. G. Anderson: "A simulation study of some dynamic assignment algorithm in a high capacity mobile telecommunication system", IEEE Trans. On Comm., Vol. COM-21, No. 11, Nov. 1973, pp. 1294-1301.

[4] S. M. Elnoubi, R. Singh, and S. C. Gupta: "A new frequency channel assignment algorithm in high capacity mobile communication systems", IEEE Trans. Veh. Technol., Vol. VT-31, No. 3, pp. $125-$ 131, Aug. 1982.

[5]Y. Furuya and Y. Akaima: "Channel segregation, a distributed channel allocation scheme for mobile communication systems", Proceedings of the 2 nd Nordic seminar on digital land mobile communications, Oct. 1986, pp. 311-315.
[6] K. Hamada, T. Ueda, and T. Otsu: "Distributer adaptive channel allocation scheme with variable $\mathrm{C} /$ threshold in cellular systems", Proceedings of thi 43rd IEEE vehicular technology conference Seacacus, 1993, pp. 164-167.

[7] H. Jiang and S. S. Rappaport: "CBWL: A new channe assignment and sharing method for cellula communications systems", IEEE Trans. On Veh Technol., Vol. VT-43, No. 2, pp. 313-322, Ma」 1994.

[8] H. Jiang and S. S. Rappaport: "A channel borrowing scheme for TDMA cellular communication systems" Proceedings of the 45th vehicular technology conference, Chicago, 1995, pp. 97-101.

[9] B. Eklundh: "Channel utilization and blocking probability in a cellular mobile telephone syster. with directed retry", IEEE Trans. Commun., vol. 34. no. 4, pp. 329-337, April 1986. 


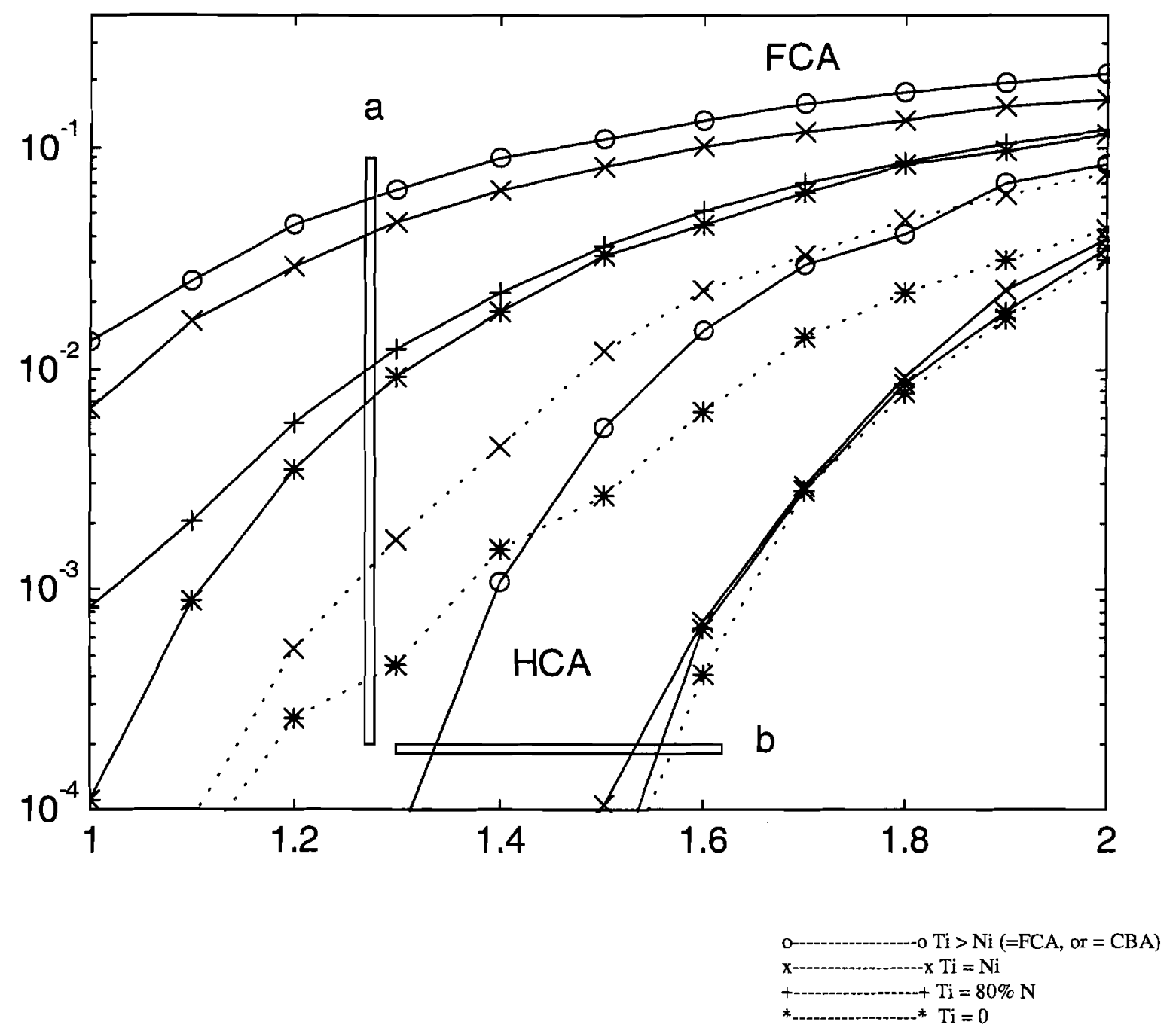

igure 5: HCA-BTV: Mean blocking probability versus traffic.

Solid lines represent no reshuffle; dashed lines represent reshuffle.

a) Dynamic-channels/fixed-channels = 0 (HCA-BTV coincides with BTV)

b) Dynamic-channels/fixed-channels $=3 / 7$ (HCA-BTV is fully implemented).

[10] M. D. Yacoub and K. W. Cattermole: "Alternative routing strategies for adjacent cells in mobile radio systems", IEE Proc.-Commun., Vol. 142, No. 2, pp. 115-120, April 199

[11] M. D. Yacoub: "Foundations of mobile radio engineering" (CRC Press, Boca Raton, Fl, USA, 1993).

[12] A. A. Shinoda and M. D. Yacoub: "Alternative routing strategy and call reshuffle in mobile radio systems", accepted for publication in Journal of the Brazilian Telecommunications Society (Revista da SBT).

[13] A. F. Victória: "Adjacent channel interference as a function of the propagation parameters and traffic distribution in cellular mobile radio systems" (in Portuguese). M.Sc. thesis, University of Campinas, Brazil.
[14] N. F. Keffer: "Cochannel interference in fuzzy cell boundaries conditions" (in Portuguese). Ph.D. thesis, University of Campinas (to be published).

[15] A. A. Shinoda: "Channel allocation algorithms in wireless communications systems" (in Portuguese). Ph.D. thesis, University of Campinas (to be published).

[16]H. Sekigushi, H. Ishikawa, M. Koyama, and H. Sawada: "Techniques for increasing frequency spectrum utilisation in mobile communication systems, "IEEE document CH2037-0/85/0000-0026, pp. 26-31, 1985.

Ailton Akira Shinoda- He received the B.Sc. (Eng.), the M.Sc. (Eng.) and Ph.D. (Eng) from the University of Campinas (UNICAMP) in 1986, 1993 and 1996, respectively. Since 1986 he has been working as a researcher at the R\&D Center of Telebras $(\mathrm{CPqD}$ - 
TELEBRAS). Currently involved in research in the field of traffic simulation in mobile communication systems.

Michel Daoud Yacoub - He received the B.Sc. (Eng.) and the M.Sc. (Eng.) from the University of Campinas (UNICAMP) in 1978 and 1983, respectively, and the Ph.D. from the University of Essex (England) in 1988. From 1978 to 1985 he worked as a researcher at the R\&D Center of Telebras (CPqD-Telebras) in the development of the Brazilian Digital SPC Exchanges - The Tropico System. He joined the R\&D Department of IBM in 1985. Since 1989 he has been a professor at the School of Electrical and Computer Engineering (UNICAMP), where his research interests concentrate in mobile communications. 